\title{
Pères de la mémoire et mémoire des pères dans l'Empire à la fin du Moyen Âge et au début de l'époque moderne
}

\section{Aude-Marie Certin}

\section{OpenEdition}

\section{Journals}

Édition électronique

URL : http://journals.openedition.org/ifha/196

DOI : $10.4000 /$ ifha. 196

ISSN : 2198-8943

\section{Éditeur}

IFRA - Institut franco-allemand (sciences historiques et sociales)

\section{Édition imprimée}

Date de publication : 6 février 2011

ISSN : 2190-0078

\section{Référence électronique}

Aude-Marie Certin, «Pères de la mémoire et mémoire des pères dans l'Empire à la fin du Moyen Âge et au début de l'époque moderne », Revue de l'IFHA [En ligne], 3 | 2011, mis en ligne le 19 avril 2012, consulté le 19 avril 2019. URL : http://journals.openedition.org/ifha/196 ; DOI : 10.4000/ifha.196

Ce document a été généré automatiquement le 19 avril 2019

(CIFHA 


\title{
Pères de la mémoire et mémoire des pères dans l'Empire à la fin du Moyen Âge et au début de l'époque moderne
}

\author{
Aude-Marie Certin
}

\section{NOTE DE L'ÉDITEUR}

Aude-Marie Certin est bénéficiaire d'une aide à la mobilité à l'IFHA depuis le 1er septembre 2011. Elle a été lauréate de la bourse Robert-Mandrou en 2010.

1 «Le souvenir des aïeux, de leurs gestes, de leur patrimoine et de leurs alliances est une constante, une sorte d'archétype inhérent à la nature humaine. Les modalités de la transmission de cette mémoire collective varient, toutefois, selon les temps et les espaces ${ }^{1}$ .» Dans l'Empire, les textes porteurs de la mémoire familiale apparaissent à partir du milieu du XIVe siècle et constituent généralement l'œuvre de pères qui s'y affirment comme tels, et s'y consacrent avec soin, parfois pendant plusieurs années. Comme l'écrit Gregor Rohmann: "C'est le père de famille, l'homme de la maison, qui écrit le livre de famille pour ses enfants et les enfants de ses enfants. De manière idéaltypique, le soin de tenir ce livre de famille est transmis au fils au moment de l'héritage de la gestion des papiers de famille².» Qu'il s'agisse de livres de famille, de livres de lignage blasonnés et costumés ou d'autobiographies, bon nombre de ces textes sont ainsi écrits par des pères soucieux de retracer les origines de leur lignage, d'évoquer la mémoire de leurs ancêtres ou de noter les informations importantes relatives à la vie de leur famille ou à leur parcours personnel ${ }^{3}$. Cependant, les pères ne sont pas seulement porteurs de la mémoire, ils sont aussi objets de la mémoire. En somme, ces sources, que l'on compte en grand nombre à la fin du Moyen Âge, révèlent l'émergence d'enjeux inédits touchant au lien 
entre paternité et mémoire dans les villes allemandes à cette époque. C'est sur cette problématique que nous aimerions apporter ici quelques éclairages.

2 Dans les années 1990-2000, d'importantes études ont porté en France sur ce thème de la paternitét ${ }^{4}$ L'anthropologie historique a notamment montré que la définition du père, comme celle de ses droits, de ses devoirs ou de ses fonctions, était une chose éminemment culturelle, qui pouvait admettre des sens fort différents selon les époques. Et la spécificité du Moyen Âge apparaît d'autant mieux si l'on rappelle que, dans la Rome antique, le paterfamilias n'avait d'heres suus que de son gré, pouvant à la naissance d'un enfant décider de se faire père ou non, ou d'adopter un enfant n'ayant aucun lien biologique avec lui ${ }^{5}$. À la fin du Moyen Âge en revanche, la définition du père est toute autre. Avec le renforcement du pouvoir de l'Église dans la société, la paternité a été institutionnalisée, se définissant désormais dans le cadre du mariage chrétien ${ }^{6}$. Dès lors, le biologique s'étaie sur le juridique et la paternité n'est plus un choix, elle s'impose à l'homme laïc dans le cadre du mariage. De plus, elle s'inscrit dans les conceptions médiévales de la parenté, nourries de la différence hiérarchisée entre spirituel et corporel. Existe de fait au bas Moyen Âge une «hiérarchie des pères ", pour reprendre l'expression de Jérôme Baschet, qui articule la paternité parfaite de Dieu le père, celle spirituelle des clercs, celle enfin des pères charnels, incarnant la forme la plus imparfaite de paternité 7 . Mais la fin du Moyen Âge se caractérise en même temps par la valorisation de certains modèles de père. Dès le XVe siècle, le culte de Joseph promu par Jean Gerson se diffuse jusqu'en Allemagne, et les sermons des hommes d'Église, comme nombre de traités de pédagogues, de juristes ou de théologiens, insistent sur l'importance de l'éducation assurée par les pères ${ }^{8}$. Dans l'Empire, la reprise de certains textes antiques, en particulier ceux de Xénophon, d'Aristote et de Cicéron, participe enfin d'une réflexion collective sur le rôle du père dans la famille et la cité. Émerge ainsi à la fin du XVe siècle un idéal de bons pères bons gouvernants ${ }^{9}$, quelques décennies avant que la Réforme ne consacre l'autorité du père dans la famille et la société ${ }^{10}$.

Depuis les années 2000, un certain nombre de travaux allemands portant sur les livres de famille, de maison ou de lignage ont aussi relevé l'importance du père de famille à la fin du Moyen Âge et au début de l'époque moderne. Différents auteurs ont ainsi insisté sur la dimension proprement paternelle de ce geste d'écriture ${ }^{11}$, montrant qu'il relevait du devoir du Hausvater de bien gérer son foyer en notant consciencieusement les événements importants de la vie de la famille (naissances, mariages, décès, transactions, comptes du ménage ...), et ce conformément à l'idéal antique de l'« économique », au sens premier du terme, tel qu'il est repris à la fin du Moyen Âge. Sur ce point, l'influence de certains ouvrages des XIVe et XVe siècles portant sur la famille ou sur le rôle du père dans celle-ci, est à noter ${ }^{12}$. Pensons par exemple au Della famiglia d'Alberti, dont Birgit Studt ${ }^{13}$ ou Christian Kuhn ${ }^{14}$ ont suggéré l'importance pour comprendre les écrits de famille allemands. En outre, l'attention des historiens allemands s'est portée sur les représentations du père dans la famille et sur la portée culturelle de la paternité à l'œuvre dans ces sources. Car l'étude des livres de famille et de lignage, comme des lettres échangées entre pères et fils, se révèle riche d'enseignements dans la perspective d'une histoire de la famille, pour étudier par exemple le rôle essentiel des pères dans l'éducation de leurs fils au cours de leurs années de formation à l'étranger ${ }^{15}$. Et cette question de la paternité a aussi été étudiée dans l'optique d'une histoire culturelle, notamment à travers l'étude des analogies rapprochant l'organisation formelle ou certains motifs de ces écrits de famille et les textes bibliques ${ }^{16}$. En somme, tous ces 
travaux récents ont révélé l'intérêt de cette question, invitant à l'étudier de façon approfondie et sous tous ces aspects conjointement.

\section{Les pères écrivains}

Dans l'Empire, ces écrits de famille ou ces textes à dimension autobiographique ont été particulièrement nombreux entre la seconde moitié du XIVe siècle et le milieu du XVIe siècle. L'une des principales caractéristiques de ces sources à cette époque touche à leur grande diversité formelle ${ }^{17}$. Souvent, en effet, les informations relatives à la vie familiale ou personnelle de leurs auteurs se trouvent mêlées à d'autres types d'informations, liées à l'histoire de leur ville ou à leurs comptes financiers. De ce fait, si l'on peut proposer une typologie de ces textes distinguant les livres de maison, les livres de famille ou de lignage et les textes à dimension autobiographique, on doit en même temps garder à l'esprit que ces textes mélangent généralement différentes formes d'écriture, et qu'il s'agit là justement d'une de leurs spécificités à cette époque ${ }^{18}$. Cette diversité s'observe en outre dans la nature même de ces entreprises d'écriture. Car si certains chroniqueurs continuent le livre de leur père, d'autres prennent l'habitude, dès leur jeunesse, de noter les événements importants de leur vie personnelle ou professionnelle, quand pour d'autres encore, l'œuvre mémorielle constitue un projet rétrospectif. Notons par ailleurs que bon nombre de ces chroniqueurs rédigent plusieurs types de livres au cours de leur vie. Le patricien de Francfort Bernard Rohrbach (1446-1482) entreprend ainsi, en 1478, la rédaction d'une première chronique intitulée Stirps Rohrbach, qui retrace les origines et l'ascension de son lignage sur cinq générations; puis il rédige un second livre, le Liber gestorum (Livre des hauts faits), dont le texte original, aujourd'hui perdu, devait compter 70 pages environ et mêler des observations personnelles et historiques, à situer entre 1338 et 1482. Quant à Matthäus Schwarz (1497-1562), directeur financier de la grande firme des Fugger d'Augsbourg, il laisse un véritable corpus documentaire, constitué de documents textuels et iconographiques divers ${ }^{19}$. À 23 ans, il rédige un premier texte intitulé Le Cours du monde (Der Weltlauf), proposant d'y décrire « tout ce qui lui était arrivé autrement qu'il l'aurait voulu ». Puis il écrit en 1518, à l'âge de 25 ans, un Traité de pratique de comptabilité ( Die Musterbuchhaltung), présentant une comptabilité modèle dans laquelle il reprend, en dépit de ses dires, son expérience comptable dans la firme des Fugger à Venise. Deux ans plus tard, en 1520, il se consacre à un genre tout à fait différent, rédigeant un Livre de prières (Gebetbuch), avant de s'intéresser, en 1524, à la généalogie biblique, dessinant à cette occasion un arbre généalogique orné de miniatures que reprendra son fils en 1533. Après 1540, Matthäus Schwarz rédige deux recueils (aujourd'hui perdus) où ses enfants tiennent une place importante : le premier rassemble des histoires pour enfants, l'autre constitue un recueil de lettres échangées avec ses fils en Italie. En parallèle, il rédige son célèbre Livre des costumes (Trachtenbuch), où il se fait représenter chaque année, à sa date anniversaire, depuis son enfance jusqu'à ses années de vieillesse. Et Matthäus Schwarz a également laissé d'autres représentations iconographiques de lui-même, que ce soit dans certaines miniatures du Livre de prières, ou dans la fameuse Danse des générations ( Geschlechtertanz) qu'il fait réaliser par le peintre Narziss Renner en 1522. Matthäus Schwarz y apparaît dans la danse des grandes familles d'Augsbourg, dans laquelle on peut également reconnaître son patron Jacob Fugger et son père Ulrich Schwarz. C'est d'ailleurs son père qui avait initié cette tradition iconographique de représentation de soi, en faisant réaliser, par Hans Holbein l'Ancien, un retable le représentant entouré de 
ses trois épouses successives et de ses 29 enfants, dont Matthäus Schwarz lui-même alors âgé de onze ans. Notons enfin que le fils aîné de Matthäus Schwarz, Veit Konrad, prend le relais de cette tradition en composant lui-même son propre Trachtenbuch en $1561^{20}$. Si cet exemple des Schwarz est assez exceptionnel par l'abondance et la diversité de ces sources textuelles et iconographiques, il apparaît néanmoins que l'entreprise de mémoire ne se limite généralement pas à un seul ouvrage et constitue même souvent une œuvre collective, réalisée par différents membres de la famille sur plusieurs générations. Ainsi, il n'est pas rare de trouver dans certaines familles de véritables ensembles documentaires comptant de nombreux documents de types différents - livres de raison, livres de famille ou de lignage blasonnés, ou notes à dimension autobiographique. Ceci s'observe par exemple dans les grandes familles patriciennes de Nuremberg comme les Tucher, les Behaim ou les Haller ${ }^{21}$. Plus que dans une œuvre mémorielle unique, reprise de façon identique de génération en génération, c'est ici dans l'accumulation de ces différentes archives que se construit et s'affirme véritablement la conscience familiale dans le temps.

Cette diversité amène à s'interroger sur les circonstances, les motifs, comme sur la portée de ces entreprises d'écriture. De fait, il n'est pas toujours possible de déterminer avec précision les circonstances ayant poussé ces hommes à prendre des notes sur leur propre vie ou à rassembler, à un moment donné de leur vie, les informations relatives à l'histoire de leur famille. Cela étant, on constate bien souvent qu'il s'agit pour eux d'affirmer l'identité de leur groupe familial et de légitimer son statut social et politique. Aussi ces chroniqueurs mettent-ils généralement en valeur la destinée glorieuse de leurs ancêtres, les alliances prestigieuses du passé, ainsi que la force de leur parenté. Pensés, élaborés et conservés par ces pères, ces textes rassemblent ainsi en un espace unique toutes les informations permettant d'assurer la prospérité de la famille. Et ce souci apparaît particulièrement important dans certains contextes sociaux et politiques. Les Familienbücher et les Geschlechtbücher se comptent en effet en grand nombre dans les villes d'Empire du Sud comme Nuremberg, Augsbourg ou Francfort, et cette corrélation n'est pas fortuite ${ }^{22}$. Car leurs auteurs, patriciens, marchands ou hommes de lettres, appartiennent aux élites de ces villes, en somme à ce milieu tout à la fois familier des livres de compte du monde marchand et des formes d'écriture officielle émanant du conseil de la ville. Par ailleurs, la production de ce type de textes s'inscrit dans le mouvement d'affirmation et de fermeture des élites urbaines ${ }^{23}$, d'où le souci de certains pères de justifier l'appartenance de leur famille au cercle étroit du patriciat en montrant l'ancienneté de leurs origines, leur honorabilité et leur souci du bien commun. La Statuslegitimation constitue en somme une motivation fréquente de ces pères-écrivains ${ }^{24}$. Mais il apparaît aussi que ces écrits sont souvent arrangés dans le but de servir de preuves dans des démarches juridiques ou politiques potentielles à venir. Là réside une autre fonction des pères, celle de noter toutes les informations relatives aux héritages, mariages ou transactions susceptibles d'aider leurs descendants à défendre leurs intérêts et ceux de leur famille en cas de conflits ${ }^{25}$. De ce fait, c'est également sur fond de silences et de non-dits que doivent se comprendre les événements choisis par ces pères pour construire la mémoire de leur famille. Tournée vers la collectivité familiale, celle-ci apparaît comme une mémoire utile, tant dans ses ambitions que dans son contenu ${ }^{26}$. Dans certaines sources enfin, le souci de transmettre la mémoire familiale et d'assurer par là même une continuité témoigne d'une inquiétude quant à l'avenir, et ce notamment lorsque la mémoire, voire l'existence même de la famille, est menacée. En témoigne par exemple le livre de famille de Werner Overstolz de Cologne, qui cherche à fixer la 
mémoire familiale dans un contexte difficile où les évolutions sociales et politiques contribuent, depuis la fin du XIVe siècle, au déclin des anciens lignages de la ville - dont celui des Overstolz - et où la famille elle-même est menacée de s'éteindre du fait du manque d'héritier. Ainsi n'est-il pas rare que la prospérité ou la respectabilité que donnent à voir ces sources visent en réalité à dépasser une situation critique pour le groupe familial ou pour certains de ses membres. Pensons ici également à Nikolaus Muffel ${ }^{27}$ (1410-1469), patricien et marchand de Nuremberg, qui écrit son Gedenkbuch deux mois avant d'être pendu pour trahison envers la ville et pour trafic d'argent, le 28 février 1469 ; compte tenu de ce contexte d'écriture, ce Gedenkbuch apparaît comme une parole de justification adressée à ses descendants, dans le but de défendre les intérêts futurs de la famille. Par leurs écrits de famille ou leurs notes personnelles, bon nombre de ces pères cherchent ainsi à transmettre un héritage stable et constructif, sur lequel leurs descendants pourront se baser pour assurer, à leur suite, la prospérité de leur famille dans un contexte d'importants changements sociaux, politiques et religieux.

\section{Fixer la mémoire, un devoir des pères}

6 Si différents soient-ils, ces textes ne sont pas pour autant sans points communs en ce qui concerne leur élaboration, leur organisation et leur contenu. En premier lieu, il convient de revenir sur le fait que cette tâche de fixer et de transmettre la mémoire incombe idéalement au père de famille. Chaînon entre ses ancêtres et ses descendants, celui-ci doit à la fois se faire le porteur de la tradition dans l'intérêt des générations futures, mais aussi un continuateur de cette même tradition, en l'enrichissant des acquis de sa propre expérience. Comme l'a montré Christiane Klapisch-Zuber pour les livres de famille florentins du XVe siècle, «l'organisation de la mémoire est un véritable devoir de tout chef de famille responsable, [auquel il revient de] transmettre le reçu, d'une part, formaliser l'acquis, d'autre part $\aleph^{28}$. Et cette double exigence se retrouve dans bon nombre des écrits de famille de l'aire germanique, ce dont témoigne d'ailleurs leur structure même. Généralement, les chroniqueurs commencent leurs écrits en reprenant les écrits et les récits de leurs prédécesseurs, puis se centrent sur leur vie propre, avant de donner la liste de leurs enfants ${ }^{29}$. Cette structure se retrouve par exemple dans la chronique de Bernard Rohrbach ${ }^{30}$ déjà évoquée. Dans les premières pages, le patricien rassemble les informations contenues dans les écrits de son grand-père et de son père, et reprend alors précisément les demandes de prières pour l'âme de ses ancêtres, ainsi que les rentes et les alliances du passé. Puis, dans les parties suivantes, Bernard Rohrbach se centre sur sa vie propre, notant ses demandes de prières pour l'âme, les informations relatives à sa famille et les grandes dates de sa vie sociale, qu'elle soit liée à ses activités dans les cercles patriciens ou au conseil de la ville. De la même façon, dans les notes autobiographiques du marchand d'Augsbourg Lucas Rem (1481-1541) ${ }^{31}$, datant de la première moitié du XVIe siècle, une première partie est consacrée aux mariages, affaires commerciales et héritages de l'arrière-grand père, du grand-père et du père de l'auteur, tandis que les neuf parties suivantes portent sur la propre vie du marchand, ce tant dans le domaine des voyages, des affaires que de la famille. Notons enfin que ces livres de famille, en constituant une prérogative des pères, marquent nettement dans le champ de la mémoire la séparation entre hommes et femmes; rares sont ainsi celles qui interviennent dans les écrits de leur mari. 
7 Du fait de leur importance symbolique, ces textes suivent le plus souvent des règles de transmission en ligne masculine avec passage aux héritiers directs ${ }^{32}$. Lazarus Holzschuher, membre d'un des lignages les plus puissants et les plus anciens de Nuremberg, est sur ce point explicite. Comme il l'écrit dans sa chronique en $1509^{33}$, il souhaite que celle-ci soit conservée par ses héritiers masculins, tout en chargeant ses fils, et plus spécialement son fils aîné, de la continuer, en inscrivant tous les noms des Holzschuher hommes et femmes, avec ceux de leur épouse ou mari, de sorte que les descendants de la famille puissent en garder mémoire. Comme en Toscane, « le fils aîné a la charge [après son père] de conserver livres et écritures, dont les plus anciens, par conséquent, restent normalement dans la branche aîné du lignage ${ }^{34}$. Ce principe s'observe également dans l'Empire, où certaines sources sont continuées de père en fils sur plusieurs générations. Chez les Behaim ${ }^{35}$ ou les Bayr ${ }^{36}$ de Nuremberg, on compte ainsi trois ou quatre générations de chroniqueurs qui se succèdent dans un même ouvrage. De même, la plupart des livres de lignage blasonnés du XVIe siècle sont poursuivis sur plusieurs générations jusqu'au XVIIe siècle, voire au-delà. Cependant, la transmission patrilinéaire des écrits de famille ne signifie pas nécessairement que le fils continue le livre de son père ou de son grand-père. Bien souvent, il arrive que le fils reprenne davantage le geste d'écriture de son père ou de ses aïeux, que leur livre même. Nombreux sont ainsi ceux qui choisissent d'écrire leur propre livre, dans lequel ils évoquent, reprennent, voire recopient les écrits de leurs prédécesseurs, tout en donnant à leur texte une forme spécifique. L'entreprise d'écriture relève en somme de l'imitation d'un père ou d'un grand-père, et constitue en soi une forme d'héritage. Dans un esprit proprement médiéval, le geste d'écriture ne saurait même avoir de véritable légitimité sans cette reprise d'un geste initié par d'autres et cette inscription dans une longue chaîne de pères, porteurs de la mémoire du groupe. Prendre sa plume pour fixer la mémoire s'inscrit dans une continuité, une forme de filiation aux ancêtres, d'où le geste d'écriture tire sa légitimité et son autorité.

8 Comme l'a montré G. Rohmann, c'est aussi le travail d'écriture des pères-écrivains au quotidien qu'il convient d'étudier ${ }^{37}$. Car le discours mémoriel de ces pères tient aussi sa force des témoignages oraux et écrits, souvent décrits comme "warhafftig " (véritables, véridiques), sur lesquels ils se basent ${ }^{38}$. Dans la chronique familiale qu'il écrit en 1523 , Albrecht Dürer (1471-1528) reprend par exemple les notes de son père portant sur la naissance de ses enfants, les recopiant mot pour mot dans sa chronique, «wie er dass in seim Buch geschrieben hat, von Wort zu Wort $~_{39}$. Quant à Lucas Rem, il fait référence dans ses premières notes aux manuscrits (" handtschriften ») de son grand-père ${ }^{40}$, puis dit noter ce que son père lui a raconté (" [das, was er] von seim vatter selig gehort ha[t]») $)^{41}$. Bien souvent, les premières pages de ces textes sont ainsi une compilation de la mémoire familiale écrite et orale ${ }^{42}$, suivant par là même "un principe médiéval d'appropriation de la mémoire antérieure", pour reprendre les termes de Pierre Monnet ${ }^{43}$. En parallèle, certains chroniqueurs mènent aussi un long travail de recherches afin de rassembler les documents importants relatifs à l'histoire de leur famille. Dans sa chronique, Lazarus Holzschuher ${ }^{44}$ évoque ainsi les documents, divers et nombreux, qu'il a rassemblés avec soin pendant sept ans. Pour « décrire comment ses ancêtres ont vécu et sont morts, et ce qui s'est passé à leur époque », le patricien se fonde sur des papiers de famille conservés par les Holzschuher eux-mêmes, sur des lettres et des livres possédés par d'autres grandes familles de Nuremberg comme les Ebner, les Grundherr ou les Pfinzing, ainsi que sur d'autres documents se trouvant dans des monastères ; autant d'archives sur lesquelles 
Lazarus Holzschuher dit s'appuyer pour prouver l'ancienneté et l'honorabilité de sa famille, et légitimer son appartenance au cercle étroit des familles patriciennes. De façon comparable à ce que l'on observe chez les Tucher, la légitimation politique passe ici par la construction d'une mémoire familiale à laquelle le poids des archives vise à donner force et autoritée 45 .

9 La portée du geste mémoriel paternel ne saurait toutefois pleinement se comprendre sans que soient évoqués les destinataires de ces écrits. Bon nombre de chroniqueurs justifient en effet leur entreprise d'écriture par le souci de s'adresser à leurs « descendants" (" Nachkommen ») ou leurs « héritiers» («Erben»). Christoph Sulzer d'Augsbourg, auteur en 1540 d'un livre de lignage, veut ainsi donner à voir à ses descendants " comme dans un miroir [...] les vertus et les actions honorables » des hommes du passé, ainsi que leurs mauvaises actions, de sorte qu'ils puissent suivre les premières et se détourner des secondes. Après avoir évoqué le modèle originaire de Dieu et des alten vetter, les Pères de la Bible, qu'il nomme un a un, l'auteur précise que ses descendants doivent avoir devant les yeux « les origines et la vie de leurs pères comme un modèle $»^{46}$. Par ces métaphores du miroir tendu aux descendants et de la double vie possible de l'homme, ce texte prend, comme la vita de l'empereur Charles IV deux siècles plus tôt ${ }^{47}$, une dimension exemplaire ${ }^{48}$. L'écriture mémorielle vise à transmettre un modèle de vie incarné par les pères du passé, afin que les descendants puissent orienter leurs actions à l'avenir. Se révèle ici l'un des enjeux essentiels de cette mémoire paternelle, à savoir l'enchaînement des générations permis par la transmission d'un certain imaginaire de la paternité.

\section{La paternité, une structure portante de la mémoire}

10 Car de fait, les pères ne sont pas seulement porteurs de la mémoire, ils constituent aussi, dans bon nombre de ces textes, une structure portante de la mémoire. Dans la chronique de Bernard Rohrbach par exemple, où cinq générations de pères sont évoquées, les pères constituent le référent prédominant par rapport auquel s'organisent toutes les parties de la chronique, qu'elles soient consacrées aux demandes de prières pour l'âme, aux rentes ou à la naissance des enfants. Et ceci se retrouve dans le livre commencé en 1488 par Michel Behaim ${ }^{49}$ de Nuremberg, et continué par la suite par l'un de ses fils puis l'un de ses petits-fils au XVIe siècle. Dans cette source, ce sont au final neuf générations de pères qui sont évoquées sur trois siècles (de la fin du XIIIe siècle à la fin du XVIe siècle) avec, pour chaque génération et de façon cyclique, la notification des naissances, des mariages et de la mort des membres de la famille ${ }^{50}$. Dans ces deux textes, comme dans bien d'autres, le poids de la paternité relève d'une certaine conception de la génération ${ }^{51}$ à la fois synchronique et diachronique: synchronique d'une part, car ces chroniques sont structurées, d'un point de vue formel, par la paternité d'un homme à chaque génération ${ }^{52}$ ; diachronique d'autre part, parce que la dynamique de la mémoire repose sur l'enchaînement des pères qui, de génération en génération, assurent la perpétuation de la famille par les naissances, les alliances et la transmission de la mémoire du groupe. Se joue ainsi dans cette chaîne des pères une idée complexe de la génération, pensée comme un fondement de la continuité et de l'unité de la famille. En mettant en jeu ces différents sens de la génération, la paternité apparaît comme un élément central, à la fois structurant et dynamique, de la mémoire familiale.

11 Mais les pères sont aussi objets de la mémoire, dans la mesure où ces textes donnent à voir des images de père, élaborant par là même une certaine conception du lien entre 
famille et société. Chez les patriciens de Nuremberg, d'Augsbourg et de Francfort, les livres de famille ou de lignage visent en effet à transmettre l'image de pères travaillant, de génération en génération, à la prospérité de leur foyer comme de leur ville. Se retrouve dans ces textes un idéal de patriciens incarnant le bon régime de la cité du fait de leur ancienneté, de l'exemplarité de leurs ancêtres et de leur souci de la chose commune, tel qu'on le trouve élaboré à la même époque dans les textes d'Aeneas Silvio Piccolimini, dans la chronique de Nuremberg de Meisterlin de 1488, ou encore dans l'éloge de cette même ville fait par Conrad Celtis dix ans plus tard ${ }^{53}$. Suivant un modèle hérité de l'Antiquité, ces livres de famille ou de lignage donnent ainsi à voir de bons pères bon gouvernants assurant, de génération en génération, la perpétuation, la prospérité et l'harmonie communes de leur maison comme de leur ville. L'on voit ici combien la famille ne saurait se comprendre comme un espace privé, strictement séparé de l'espace social et politique. Selon l'expression de Simon Teuscher, «les chroniques familiales fournissent des possibilités uniques d'appréhender la spécificité des liens existant, à la fin du Moyen Âge, entre famille, parenté et pouvoir municipal »; aussi s'agit-il d'analyser ces écrits comme des « instruments à usage familial et politique ${ }^{54}$.

Pour autant, l'analyse de sources émanant d'autres milieux révèle que l'articulation entre paternité, famille et société peut prendre des formes différentes, et posséder d'autres enjeux. Si l'on s'intéresse aux notes autobiographiques de certains marchands, il apparaît en effet que le discours mémoriel est davantage centré sur le vécu quotidien du père dans sa famille. Dans les notes de Lucas Rem, par exemple, l'évocation du père charnel comme la référence aux pères du passé sont assez succinctes; en revanche, le marchand fait de sa propre paternité un élément fort de son identité sociale, à côté de ses activités marchandes. Parallèlement à ses notes consacrées à ses voyages d'affaire, ses comptes financiers et ses listes de dépense, Lucas Rem consacre ainsi deux parties à ses enfants, dans lesquelles il fait un portrait physique et moral de chacun d'eux. Et le discours sur la vie quotidienne de la famille et l'éducation prodiguée par le père à ses enfants donne à voir un idéal moral, où le père de famille joue un rôle central. Ces notes de Lucas Rem, comme l'autobiographie de Burkard Zink (1396-1474/75) écrite entre 1460 et 1468, montrent ainsi que pour ces marchands, l'honorabilité sociale est corrélative d'une certaine image du père, assurant la prospérité de sa famille comme de sa société de commerce, révélant par là même sa moralité et son élection divine.

Dans les écrits des humanistes, le discours sur le père de famille est tout aussi prégnant, mais il prend sens à l'aune d'autres référents culturels. La chronique d'Albrecht Dürer, par exemple, a pour objet principal le propre père du peintre. Comme il l'écrit d'ailleurs explicitement dans les premières lignes, « moi, Albrecht Dürer le Jeune, j'ai recueilli dans les écrits de mon père et réuni ces renseignements sur son origine, sur sa venue, son existence ici et sur sa pieuse fin $\aleph^{55}$. Après avoir raconté les origines de son père, Albrecht Dürer évoque ainsi le soin que celui-ci portait à l'éducation de ses enfants et son souci de leur parler quotidiennement afin qu'ils apprissent à être bons envers Dieu et envers les hommes $^{56}$. Au fil de cette chronique se dessine peu à peu le portrait d'un père tout à la fois honorable, profondément pieux et soucieux de ses enfants - en somme une figure modèle de père, telle qu'on la trouve élaborée à la même époque dans la pensée humaniste, dont Albrecht Dürer était familier, ainsi que dans certains traités d'éducation, comme Le Livre du chevalier de La Tour Landry pour l'enseignement de ses filles (1371-1372) ${ }^{57}$, traduit en allemand sous le titre Ritter von Turn (1493) et dont Albrecht Dürer fit les illustrations ${ }^{58}$. Dans la chronique familiale du peintre, comme dans la vita de son ami 
humaniste Willibald Pirckheimer, la paternité est ainsi un thème central. Et si père et enfant sont envisagés de façon plus individuelle dans la sphère de la famille, ce discours sur la relation père-enfant reste en même temps porteur d'un idéal social d'harmonie collective dans la cité.

\section{Paternité et dynamiques de la mémoire}

14 Les livres de famille ou les textes autobiographiques écrits dans l'Empire à la fin du Moyen Âge et au début de l'époque moderne révèlent combien la paternité est un enjeu essentiel de la mémoire à cette époque. Idéalement, ce sont en effet les pères qui portent la mémoire, la structurent et lui donnent sens. En d'autres termes, la paternité tient une place déterminante à tous les niveaux de l'écriture mémorielle, celle-ci étant à la fois un ensemble de pratiques, mêlant gestes, mots et images, revenant idéalement au père de famille ; un dispositif formel, structuré et scandé par certains motifs, en l'occurrence par une chaîne des pères; et un dispositif culturel participant de la définition des réalités sociales, à travers les images du père qui y sont élaborées. Par là même, la mémoire donne aux pères une importance inédite dans la famille et la cité, faisant d'eux les garants de l'identité du groupe dans le temps. Porteurs et objets de la mémoire, les pères ont ainsi pour fonction d'assurer l'enchaînement des générations passées, présentes et à venir, dans un contexte d'importants changements sociaux, politiques et confessionnels. Et dans cette pensée mémorielle, la famille et la cité apparaissent comme deux sphères intimement liées, dont la longévité et la prospérité communes reposent sur la perpétuation de certaines images paternelles que ces écrits visent tout à la fois à façonner et à transmettre. Cette pluralité des images du père est importante à relever car, comme l'écrit Jean-Claude Schmitt, il convient de réfléchir à « la culture médiévale en terme de pôles multiples et de relations complexes, d'une façon plus proche des réalités concrètes du tissu social : il n'y a pas une culture ecclésiastique, [...] ni une culture urbaine, mais des théâtres locaux d'ambition et de conflits qui mettent en jeu tel ou tel segment de la société $"{ }^{59}$. Aussi quand, pour les membres des anciennes élites urbaines, l'enjeu est d'assurer la perpétuation d'un ordre social et politique dominé par les " patriciens », pour d'autres, marchands ou humanistes, l'enjeu du discours sur le père est plutôt d'affirmer une autre idée de l'honorabilité sociale ou un idéal éducatif ${ }^{60}$. Ainsi ces textes donnent-ils à voir différents modèles reliant paternité, famille et société qui portent et révèlent, par leurs tensions mêmes, les dynamiques sociales à l'œuvre à cette époque. Pour autant, si différentes que puissent être leurs orientations, l'ensemble de ces textes témoigne du fait que cette articulation proprement culturelle entre mémoire et paternité constitue une composante déterminante de la redéfinition du jeu social dans l'aire germanique entre le milieu du XIVe siècle et l'époque de la Réforme. 


\section{NOTES}

1. Martin Aurell, «Introduction. Modernité de la monographie familiale » in : Id. (dir.), Le Médiéviste et la monographie familiale : sources, méthodes, problématiques, Turnhout : Brepols, 2004, p. 7.

2. Gregor Rohmann, Eines Ehrbaren Rathts gehorsamer amptmann. Clemens Jäger und die Geschichtsschreibung des 16. Jahrhunderts, Augsburg: Wissner, 2001, p. 142. Sur ce sujet voir aussi Id., Das Ehrenbuch der Fugger. Darstellung-Transkription-Kommentar, vol. 1, Augsburg : Wissner, 2004, p. 25-38.

3. Pour une présentation de ces sources, voir Helmut Haller von Hallerstein, " Nürnberger Geschlechtsbücher » in : Mitteilungen des Vereins für Geschichte der Stadt Nürnbergs, 65, 1978, p. 134-147 ; Urs-Martin Zahnd, Die autobiographischen Aufzeichnungen des Ludwig von Diesbachs. Studien zur spätmittelalterlichen Selbstdarstellung im oberdeutschen und schweizerischen Raume, Bern: Berner Burgerbibliothek, 1986, p. 279-397 ; Pierre Monnet, Les Rohrbach de Francfort. Pouvoirs, affaires et parenté à l'aube de la Renaissance allemande, Genève: Droz, 1997; G. Rohmann, Eines Ehrbaren Rathts gehorsamer amptmann, op. cit.; Barbara Schmid, Schreiben für Status und Herrschaft. Deutsche Autobiographik im Spätmittelalter und früher Neuzeit, Zürich : Chronos, 2006.

4. Didier Lett (dir.), Être père au Moyen Âge. Cahiers de recherches médiévales et humanistes, 4, 1997 ; Jérôme Baschet, Le Sein du père. Abraham et la paternité dans l'Occident médiéval, Paris : Gallimard, 2000 ; Jean Delumeau, Daniel Roche (dir.), Histoire des pères et de la paternité, Paris : Larousse, 2000 ; Paul Payan, Joseph. Une image de la paternité dans l'Occident médiéval, Paris : Aubier, 2006.

5. Jacques Mulliez, «La désignation du père » in : J. Delumeau, D. Roche (dir.), Histoire des pères et de la paternité, op. cit., p. 43-72 ; Yan Thomas, « À Rome, pères citoyens et cité des pères » in : André Burguière, Christiane Klapisch-Zuber et alii (dir.), Histoire de la famille, Paris : A. Colin, 1986, p. 253-302.

6. Anne Lefebvre-Teillard, «Pater is est quem nuptiae demonstrant. Jalons pour une histoire de la présomption de paternité " in: Revue d'histoire de droit français et étranger, 69/3, 1991, p. 340.

7. J. Baschet, Le Sein du père, op. cit., p. 331-343.

8. D. Lett, L'Enfant des miracles. Enfance et société au Moyen Âge (XIIe-XIIIe siècles), Paris : Aubier, 1997, notamment p. 149-151; Eugenio Garin, «L'image de l'enfant dans les traités de pédagogie du XVe siècle » in : Egle Becchi, Dominique Julia (dir.), Histoire de l'enfance en Occident (t. I). De l'Antiquité au XVIIe siècle, Paris : Seuil, 1998, p. 247-270.

9. P. Monnet, « La mémoire des élites urbaines dans l'Empire à la fin du Moyen Âge entre écriture de soi et histoire de la cité » in : Hanno Brand, Pierre Monnet, Martial Staub (dir.), Mémoria, communitas, civitas. Mémoire et conscience urbaines en Occident à la fin du Moyen Âge, Stuttgart : J. Thorbecke, 2003, p. 52-56.

10. Marianne Carbonnier-Burkard, «Les variations protestantes » in: J. Delumeau, D. Roche (dir.), Histoire des pères et de la paternité, op. cit., p. 169-191. 
11. Birgit Studt, «Erinnerung und Identität. Die Repräsentation städtischer Eliten in spätmittelalterlichen Haus- und Familienbüchern» in: Ead. (dir.), Haus- und Familienbücher in der städtischen Gesellschaft des Spätmittelalters und der frühen Neuzeit, Köln/ Weimar/ Wien : Böhlau, 2007, p. 1-31.

12. Sur ce sujet, voir Gotthardt Frühsorge, «Die Begründung der "väterlichen Gesellschaft" in der europäischen oeconomia christiana. Zur Rolle des Hausväterliteratur des 16. bis 18. Jahrhunderts in Deutschland» in: Hubertus Tellenbach (dir.), Das Vaterbild im Abendland, Stuttgart: Kohlhammer, 1978, p. 110-123 ; Trude Ehlert, « Die Rolle von "Hausherr" und "Hausfrau" in der spätmittelalterlichen volkssprachigen Ökonomik » in : Ead. (dir.), Haushalt und Familie in Mittelalter und früher Neuzeit, Sigmaringen: J. Thorbecke, 1991, p. 153-166; B. Studt, "Der Hausvater. Haus und Gedächtnis bei Hermann von Weinsberg » in : Rheinische Vierteljahrblätter, 61, 1997, p. $135-160$.

13. B. Studt, « Erinnerung und Identität », op. cit., p. 1-2.

14. Christian Kuhn, «Von Wohl, Ehre und Grösse der Familie zu Generation. Der Generationsdiskurs in Albertis Della Famiglia (1433-41) und in der Familiengeschichtsschreibung Christoph Scheurls (1542) » in : Mark Häberlein, Christian Kuhn, Lina Hörl (dir.), Generationen in spätmittelalterlichen und frühneuzeitlichen Städten (ca. 1250-1750), Konstanz : UVK, 2011, p. 93-115.

15. Mathias Beer, «Das Verhältnis zwischen Eltern und ihren jugendlichen Kindern im spätmittelalterlichen Nürnberg " in : Mitteilungen des Vereins für die Geschichte der Stadt Nürnberg, 77, 1990, p. 91-153, ici p. 143-153; Chr. Kuhn, Generation als Grundbegriff einer historischen Geschichtskultur. Die Nürnberger Tucher im langen 16. Jahrhundert, Göttingen : Vandenhoeck \& Ruprecht, 2010, p. 109-233.

16. G. Rohmann, Das Ehrenbuch der Fugger, op. cit., p. 109-112 ; Chr. Kuhn, Generation als Grundbegriff einer historischen Geschichtskultur, op. cit.

17. U.-M. Zahnd, "Stadtchroniken und autobiographische Mitteilungen. Studien zur Selbstdarstellung spätmittelalterlicher Bürger » in : Klaus Arnold, Sabine Schmolinsky, Urs-Martin Zahnd (dir.), Das dargestellte Ich. Studien zu Selbstzeugnissen des späteren Mittelalters und der frühen Neuzeit, Bochum : Winkler, 1999, p. 51.

18. Sur les spécificités de ces sources médiévales et la question de leur typologie, voir entre autres U.-M. Zahnd, Die autobiographischen Aufzeichnungen des Ludwig von Diesbachs, op. cit.; S. Schmolinsky, « Selbstzeugnisse im Mittelalter » in : Kl. Arnold, S. Schmolinsky, U.-M. Zahnd (dir.), Das dargestellte Ich, op. cit., p. 19-28.

19. Philippe Braunstein, Un banquier mis à nu. Autobiographie de Matthäus Schwarz, bourgeois d'Augsbourg, Paris: Gallimard, 1992, p. 102-105. Pour la présentation et l'analyse des témoignages textuels et iconographiques laissés par les Schwarz, voir aussi August Fink, Die Schwarzschen Trachtenbücher, Berlin: Deutscher Verein für Kunstwissenschaft, 1963 ; Jean-Claude Schmitt, L'Invention de l'anniversaire, Paris : Arkhê, 2009, p. 17-63.

20. J.-Cl. Schmitt, L'Invention de l'anniversaire, op. cit., p. 39-43.

21. Sur les nombreuses sources conservées pour les familles Tucher, Behaim, Haller, voir les références bibliographiques données en note 3 . 
22. P. Monnet, «La monographie familiale entre histoire urbaine et histoire culturelle : l'exemple des pays germaniques de l'Empire à la fin du Moyen Âge » in : M. Aurell. (dir.), Le Médiéviste et la monographie familiale, op. cit., p. 47.

23. P. Monnet, Les Rohrbach de Francfort, op. cit.

24. U.-M. Zahnd, Die autobiographischen Aufzeichnungen des Ludwig von Diesbachs, op. cit., notamment p. 309-335.

25. Simon Teuscher, «Parenté, politique, comptabilité. Chroniques familiales autour de 1500 (Suisse et Allemagne du Sud) » in : Annales. Histoire sciences sociales, 59, 2004, p. 858.

26. Jean Tricard, «Les livres de raison français au miroir des livres de famille italiens. Pour relancer une enquête » in : Revue historique, 307, 2002, p. 1001.

27. Carl Hegel (éd.), Gedenkbuch von Nikolaus Muffel (1468), Leipzig: Hirzel, 1874, p. 735-751.

28. Chr. Klapisch-Zuber, "Comptes et mémoire: l'écriture des livres de famille florentins » in : Caroline Bourlet, Annie Dufour (dir.), L'Écrit dans la société médiévale. Divers aspects de sa pratique du XIe au XVe siècle. Textes en hommage à Lucie Fossier, Paris : Éd. du CNRS, 1991, p. 252.

29. U.-M. Zahnd, «Einige Bemerkungen zu spätmittelalterlichen Familienbüchern aus Nürnberg und Bern » in : Rudolf Endres (dir.), Nürnberg und Bern. Zwei Reichsstädte und ihre Landgebiete, Erlangen : Universitätsbibliothek, 1990, p. 9-10.

30. Georg Eduard Steitz, "Die Familienchronik Bernhard Rohrbachs aus dem 15. Jahrhundert » in : Archiv für Frankfurter Geschichte und Kultur, II/2, 1865, p. 404-437.

31. Benedict Greiff, « Tagebuch des Lucas Rem aus den Jahren 1494-1541. Ein Beitrag zur Handelsgeschichte der Stadt Augsburg " in: 26. Jahresbericht des Historischen Kreisvereins für Schwaben und Neuburg, 27, 1861, p. 1-110.

32. Chr. Klapisch-Zuber, "Comptes et mémoire: l'écriture des livres de famille florentins », op. cit., p. 253.

33. Nuremberg, Stadtarchiv, Holzschuher, E 49/III 1, fol. 8.

34. Chr. Klapisch-Zuber, "L'invention du passé familial à Florence (XIVe-XVe siècles)" in : Temps, mémoire et tradition au Moyen Âge, Aix-en-Provence/ Marseille : J. Laffitte, 1983, p. 29.

35. Nuremberg, Stadtarchiv, E 11/II FA Behaim n507.

36. Nuremberg, Staatsarchiv, Handschriften Rep. 52 a. 266.

37. Gr. Rohmann, « Mit seer grosser Muhe und schreiben an ferre ort » in : B. Studt (dir.), Haus- und Familienbücher in der städtischen Gesellschaft des Spätmittelalters und der frühen Neuzeit, op. cit., p. 87-120.

38. U.-M. Zahnd, «Einige Bemerkungen zu spätmittelalterlichen Familienbüchern aus Nürnberg und Bern », op. cit., p. 8.

39. Hans Rupprich, Dürer. Schriftlicher Nachlass, Berlin: Deutscher Verein für Kunstwissenschaft, 1956, p. 29.

40. B. Greiff, « Tagebuch des Lucas Rem aus den Jahren 1494-1541 », op. cit., p. 1.

41. B. Greiff, « Tagebuch des Lucas Rem aus den Jahren 1494-1541 », op. cit., p. 2. 
42. Sur la question des rapports entre auteurs et autorités dans les écrits autobiographiques médiévaux, voir J.-Cl. Schmitt, La Conversion d'Hermann le Juif. Autobiographie, histoire et fiction, Paris : Seuil, 2003, p. 63-65.

43. P. Monnet, «La mémoire des élites urbaines dans l'Empire à la fin du Moyen Âge entre écriture de soi et histoire de la cité ", op. cit., p. 61.

44. Nuremberg, Stadtarchiv, Holzschuher, E 49/III 1, fol. 4.

45. U.-M. Zahnd, Die autobiographischen Aufzeichnungen des Ludwig von Diesbachs, op. cit., p. 310.

46. Augsbourg, Staatsbibliothek, $2^{\circ}$ Cod. Aug. 131, fol. 1-2.

47. P. Monnet, J.-Cl. Schmitt (éd.), Vita de Charles IV, Paris : Les Belles-Lettres, 2010, p. LII-LIII.

48. Sur cette question, voir aussi les exemples donnés par Gr. Rohmann, Das Ehrenbuch der Fugger, op. cit., p. 74.

49. Nuremberg, Stadtarchiv, E 11/II FA Behaim, n507.

50. Sur la question de la memoria dans la famille, à savoir les liens unissant les vivants et les morts, voir notamment Otto Gerhard Oexle, «Memoria als Kultur » in : Id. (dir.), Memoria als Kultur, Göttingen : Vandenhoeck \& Ruprecht, 1995, p. 9-78.

51. M. Häberlein, Chr. Kuhn, L. Hörl (dir.), Generationen in spätmittelalterlichen und frühneuzeitlichen Städten (ca. 1250-1750), op. cit.; Chr. Kuhn, Generation als Grundbegriff einer historischen Geschichtskultur, op. cit. Sur ce sujet, voir aussi les analyses de U.-M. Zahnd, Die autobiographischen Aufzeichnungen des Ludwig von Diesbachs, op. cit., p. 310-311 et de P. Monnet, Les Rohrbach de Francfort, op. cit., p. 117-120.

52. Sur ce point, voir les analyses de Chr. Kuhn à propos du livre de lignage des Tucher de Nuremberg dans Id., Generation als Grundbegriff einer historischen Geschichtskultur, op. cit., p. 95.

53. P. Monnet, «La mémoire des élites urbaines dans l'Empire à la fin du Moyen Âge entre écriture de soi et histoire de la cité », op. cit., p. 52-56.

54. S. Teuscher, « Parenté, politique, comptabilité », op. cit., p. 848. Sur cette question, il convient aussi de se référer à l'étude pionnière d'Erich Maschke, «Die Familie in der deutschen Stadt des späten Mittelalters " in: Sitzungsberichte des Heidelberger Akademie, 4, 1980, p. 9-98, ainsi qu'aux études de P. Monnet, notamment dans Les Rohrbach de Francfort, op. cit. ; Id., « Élites dirigeantes et distinction sociale à Francfortsur-le-Main » in: Francia, 27, 2000, p. 117-162; Id., "Villes et société urbaine dans l'Empire à la fin du Moyen Âge » in : BullMHFA, 39, 2003, p. 167-181.

55. «Ich, Albrecht Dürrer der jünger, hab zusammen aus meines vatters schriften, von wannen er gewesen sej, wie er herkumen und blieben und geendet seeliglich » in : H. Rupprich, Dürer. Schriftlicher Nachlass, op. cit., p. 28; Albrecht Dürer, Traité des proportions accompagné des lettres et écrits théoriques, Pierre Vaisse (éd.), Paris : Hermann, 2008, p. 41.

56. « Darumb was sein täglich sprach zu uns, dass wir gott lieb solten haben und treulich gegen unsern nechsten handeln ", in : H. Rupprich, Dürer. Schriftlicher Nachlass, op. cit., p. 30 .

57. D. Lett, « Comment parler à ses filles ? » in : Médiévales, 19, 1990, p. 77-82. 
58. P. Vaisse, Dürer, Paris : Fayard, 1995, p. 29.

59. J.-Cl. Schmitt, Le Corps, les rites, les rêves, le temps. Essais d'anthropologie médiévale, Paris : Gallimard, 2001, p. 19.

60. Ces textes articulent en somme de façon chaque fois spécifique les options « froides » et « chaudes » de la mémoire étudiées par Jan Assmann, voir J. Assmann, Das kulturelle Gedächtnis. Schrift, Erinnerung und politische Identität in frühen Hochkulturen, 1re éd., München : Beck, 1997 ; trad. fr., La Mémoire culturelle. Écriture, souvenir et imaginaire politique dans les civilisations antiques, Paris : Aubier, 2010, p. 60-71. Sur cette question, nous renvoyons aussi à Gr. Rohmann, Das Ehrenbuch der Fugger, op. cit., p. 78. 